\title{
Designing Health Data Management Systems: Learning From Prominent Worldwide Applications
}

\author{
Yu Yawen ${ }^{1,2}$, Masafumi Nakamura $^{2}$ and Naoki Nakashima ${ }^{1^{*}}$
}

${ }^{1}$ Medical Information Center, Kyushu University Hospital, 3-1-1, Maidashi, Higashi-ku, Fukuoka 812-8582, Japan

${ }^{2}$ Department of Surgery and Oncology, Graduate School of Medical Sciences, Kyushu University, 3-1-1, Maidashi, Higashi-ku, Fukuoka 812-8582, Japan

"Corresponding author: Naoki Nakashima, Medical Information Center, Kyushu University Hospital, 3-1-1, Maidashi, Higashi-ku, Fukuoka 812-8582, Japan, Tel: +81926425881; E-mail: nnaoki@info.med.kyushu-u.ac.jp

Received date: December 14, 2015; Accepted date: February 18, 2016; Published date: February 28, 2016

Copyright: (c) 2016 Yawen Y, et al. This is an open-access article distributed under the terms of the Creative Commons Attribution License, which permits unrestricted use, distribution, and reproduction in any medium, provided the original author and source are credited.

\begin{abstract}
Living in a world of data, we need to use data wisely and manage it efficiently, as well as never get lost into it, especially in the field of health care. The design of health data management systems is a critical factor.

From applications in different fields, we have chosen three that are characteristic of good data management and that may inspire better design of health data management systems: visualize data by mind mapping, track data by timeline, and increase interactivity by "check-in" function.
\end{abstract}

Keywords: Health data management; Health Care; Mind Mapping; Timeline

\section{Introduction}

Worldwide, 2.5 quintillion bytes of data are produced every day. Indeed, $90 \%$ of the data in the world today was created in the last 2 years alone [1]. We are living in the "big data" century, and we expect to make decisions based on data, especially in the field of health care. However, we still lack practical ways to realize it.

These days, we are not short of information, but we lack ways to manage it wisely. Every minute, new smartphone applications, special sensors for medical use, health care devices, etc. are launched. We can obtain data so much more easily than before [2]; despite this, we hardly know much more than before by the data management systems existing, because it can be difficult to know where to focus on.

Comparing existing health data management systems, it is common for data to burst into view on the screen after one simple click [3]. Suddenly, all the important information is available; we read it carefully and try our best not to leave any of it out. In health care, there is a trend to consider that the available data is inadequate to determine the perfect physical condition. Because of that, we have focused on gaining access to more data, but we have not concentrated on effective management of the data. However, if we cannot see anything further by effective management [3], not to mention making decisions based on the large quantity of information at our fingertips, we will fall into a vicious circle: getting lost into big health data and gaining access to more data, over and over again.

\section{Examples of effective data management}

Three distinctive and inspiring examples of effective data management provide valuable insights into the subject.

Example 1: Mind mapping. Blue Button is a program that allows users to view their personal health records online and download them
[4]. Several U.S. federal agencies have implemented this capability for their beneficiaries.

Research has shown a high self-reported impact of the Blue Button among current users: $73.3 \%$ reported that it helps them understand their health history better because all the information is in one place, $72.2 \%$ reported that it helps them monitor their laboratory results better, and $67.9 \%$ reported that it makes it easier for them to give others important information about their health [5]. Most of Blue Button's users are in the United States.

Blue Button's data visualization is one of the factors that make it stand out. It can be considered to be an application of mind mapping that shows potential in medicine information management [6]. The user's first sight of the Blue Button website is not of trivial data but of the system of records showing an overview of the available information and helping the users become aware of how the data are used [7]. Keywords and short explanations work well when users try to extract information from their personal records.

Mind mapping is a well-established teaching and learning method that involves drawing diagrams to visualize and organize information [8]. A central concept, drawn as a simple illustration, is linked with representations of associated ideas, along with images, keywords, and short explanative phrases. Each major idea is connected directly to the central concept, and sub-ideas form branches [9]. In general, mind mapping is used as a tool that helps the user connect new information meaningfully with their existing knowledge and as a memory aid [10]. Data presentation using a mind map requires the careful design of keywords, short explanations, and simple illustrations in various colors. With its unique appearance and visual appeal, mind mapping works in a similar way to the human brain when recalling and remembering information, making the information simpler to understand.

Besides, Mind mapping has been used for online health records, and for disseminating new information among patients and medical staff [11]. A study demonstrates that mind mapping can be easily taught to medical students who have no previous background in mind mapping. 
This finding suggests the strength of mind mapping even after a single, 30 -minute introductory session in promoting critical thinking in the novice learner, and supports the notion of adult learner capability [12].

Example 2: Use of a timeline. Elevate is a smartphone application that is a cognitive training tool designed to build communication and analytical skills. Members are provided with a personalized gamebased training program that is adjusted over time based on performance. It can also be seen as part of PHRPersonal Health Records system with a mobile application version [13]. Elevate can be downloaded for free on both Apple Android smartphones; this allows wide usage, but it is not easy to use by non-English-speaking individuals.

A notable feature of Elevate is that it puts different kinds of data in one timeline, and uses different colors represent brain functions trained by the application. Various kinds of data organized in colors by timeline, which ensures the user can check all the functions by date. Organizing different kinds of data independently and checking them in the timeline together makes data management more flexible. This is important because in PHRs, the timeline can range from days to months and years and even a lifetime [14].

Using the timeline to track data is another reason for building a health care management system with interactive visualization. For example, the system requires tracking daily data regarding drug dosage, pulse rate, change of weight, etc. Checking everyday data according to a natural timeline ensures the data, including health data and clinical data, are properly collected [15]. Furthermore, a timeline could be used in intensive medicine for checking a patient's available medical history from the moment the patient is admitted into the Intensive Care Unit until discharge [16].

Timeline also shows potential in the analysis of drug interactions information. Health records in timeline have good longitudinal coverage of patient history, and thus are likely to provide an accurate measure on a particular drug combination among patients. 569398 drug-drug-event combinations in a research have been published [17]. The first step of mining clinical records is to structure data in timeline.

However, to use a timeline to handle long-term data, we first need to extract the temporal relationships among the clinical records, stored as computer code and unstructured free text [18].

Example 3: Check-in function. Shanbay is not a health care application but a foreign language learning application. It has gained tens of millions of users, providing a free smartphone application download and website personal accounts. The application only has a Chinese version and is therefore almost impossible to use in other countries.

Its check-in function merits attention. The check-in record is in a conspicuous position when the user signs into the website or opens the mobile application, so the user can see it immediately. The user can check in only after finishing all the learning tasks. The user is awarded additional points by checking in day by day. Points can be exchanged for extra services. Users can also pay for points to get extra services they need. Furthermore, after checking in, users are encouraged to share their result on social networks and to friends by earning points. Hardworking users have built groups to supervise the daily check-in of each other.

In health care management, we often need to track everyday data, although this may not work in a supportive way when the data is input by the user. Interactive application with functions like "check-in" could increase the quality of health data self-management and self-control [19]. For instance, alarms could be set to remind users to input their daily record, and functions could be provided to allow health records to be shared with friends, families, doctors, and social networks.

User loyalty and interactivity is important in health care management. We have found that in self-learning applications and shopping websites, functions like "check-in" are normally used to improve the quality of their services. For instance, Duolingo, a language learning application has 10 million users around the world; Taobao, a shopping website of Alibaba Group, has 350 million active users by March 2015 [20]. They are using this kind of function to help users keep learning everyday or increase buying intention by registering the website frequently. It will enhance the health care management if we apply the functions like "check-in".

\section{Discussion}

A well-designed health care management system is not just for data collection, but also for using the large quantity of information collected to make decisions. In the "big data" century, we can never check all the details on health records. Using both logic-oriented and time-oriented methods to manage data are essential. Therefore, methods for gathering important information, such as mind mapping and the use of a timeline, which provide a good overview of all the data collected, would increase efficiency. Moreover, functions such as "check-in" may provide an opportunity to link with social networks and come onto the market.

Health care data management systems are expected to provide lifelong records, such as PHR, the electronic part of the health-related information about a person that includes diagnoses, medications, allergies, lab test results, and prescription renewals, which can be extracted from multiple sources. The use of PHR could potentially increase the efficiency of medical and health care services and reduce costs; but challenges still remain in the near future [21].

Security is one of the problems at issue. Health care data management system like PHR is a patient-centric model where it is expected that health information would be exchanged, with data possibly exposed to third-party servers such as cloud storage [22]. Another reason is that systems are not under the same security situation by using different EHR (Electronic Health Records) systems among hospitals. What's more, without standardized and unified security procedure, patient's data is possible to leak by unrelated staff in hospital.

The current health care systems are changing from professional centric to patient centric. In a well-designed system, individuals are equal to take part in managing their wellness [23]. Therefore, we need to simplify the management for public. Among applications in different fields, we found out 3 functions showing potential and inspiring, however, they have not been used together in health care management yet: using mind mapping makes management systematic and easy to understand; track data by timeline simplifies the interface and improve data readability; functions such as "check-in" hopefully increase user interactivity.

In the future research, security is always crucial in health care. Cooperate with professionals in different fields, such as medicine, engineering and law etc., would be important to build a secure system. It is also helpful in gaining crossover point of view in the study. For individual users, we focus on simplifying the interface and increasing readability, and for health care providers, data analytics is essential. 
Citation: Yawen Y, Nakamura M, Nakashima N (2016) Designing Health Data Management Systems: Learning From Prominent Worldwide Applications. J Health Med Inform 7: 216. doi:10.4172/2157-7420.1000216

Page 3 of 3

After designing a good system, using the data collected from individual users needs more attention, especially in the big data century, making decisions by analyzing the data is always fascinating. However, we still have a long way to go before seeking out more effective strategies for health care management.

\section{References}

1. Zikopoulos P (2015) Big Data beyond the Hype: A Guide to Conversations for Today's Data Center, McGraw-Hill Education.

2. Cuttone A, Lehmann S, Larsen JE (2013) A mobile personal informatics system with interactive visualizations of mobility and social interactions. PDA 13: 27-30.

3. Schouten P (2013) Big data in health care. Healthc Financ Manage 67: 40-42.

4. Vogel L (2010) "Blue button" access to medical records. CMAJ 182: 746

5. Turvey C, Klein D, Fix G, Hogan TP, Woods S, et al. (2014) Blue Button use by patients to access and share health record information using the Department of Veterans Affairs' online patient portal. J Am Med Inform Assoc 21: 657-663.

6. Gurrero J, Ramos P (2015) Applications of Mind Mapping Automation In Medicine, Introduction to the Applications of Mind Mapping in Medicine, Createspace.

7. Walker D, Adebajo A, Heslop P, Hill J, Firth J (2007) Patient education in rheumatoid arthritis: the effectiveness of the ARC booklet and the mind map. Rheumatology 46: 1593-1596.

8. Edwards S, Cooper N (2010) Mind mapping as a teaching resource, The Clinical Teacher. 7: 236-239.

9. Davies M (2011) Concept mapping, mind mapping and argument mapping: what are the differences and do they matter? High Educ 62 279-301.

10. Rosciano A (2015) The effectiveness of mind mapping as an active learning strategy among associate degree nursing students. Teaching and Learning in Nursing 10: 93-99.
11. Austin R, Hull S, Westra B (2014) Blue button movement: engaging ourselves and patients. Comput Inform Nurs 32: 7-9.

12. D'Antoni AV, Zipp GP, Olson VG, Cahill TF (2010) Does the mind map learning strategy facilitate information retrieval and critical thinking in medical students. BMC Med Educ 10: 61.

13. Murdoch TB, Detsky AS (2013) The inevitable application of big data to health care. JAMA 309: 1351-1352.

14. Stafford FP (2009) Timeline data collection and analysis: time diary and event history calendar methods. Calendar and Time Diary.

15. Grouin C, Grabar N, Hamon T, Rosset S, Tannier X, et al. (2013) Eventual situations for timeline extraction from clinical reports. J Am Med Inform Assoc 820-827.

16. Braga A, Portela F, Santos MF, Machado J, Abelha A, et al. (2015) Step towards a patient timeline in intensive care units. Procedia Computer Science, 64: 618-625.

17. Iyer SV, Harpaz R, LePendu P, Bauer MA, Shah NH (2014) Minging clinical text for signals of adverse drug-drug interactions. J Am Med Inform Assoc 21: 353-362.

18. Nikfarjam A, Emadzadeh E, Gonzalez G (2013) Towards generating a patient's timeline: Extracting temporal relationships from clinical notes. J Biomed Inform 46 Suppl: S40-47.

19. Li I, Medynskiy Y, Froehlich J, Larsen J (2012) Personal informatics in practice: improving quality of life through data. CHI EA 12: 2799-2802.

20. Smith C (2015) By the numbers: 60 amazing Alibaba statistics. DMR.

21. Krukowski A, Vogiatzaki E (2014) Adaptations of personal health record platform for medical research on chronic diseases. EAI Endorsed Transactions on Pervasive Health and Technology 15: e5.

22. Hoskery AK, Kumar V, Mathur S, Kumawat A (2015) Scalable and secure sharing of personal health records: a review. J Emerg Technologies Innov Res 2: 1809-1814.

23. Saranummi N (2013) In the Spotlight: Health Information Systems Connected Health and Information Technology for Health. Reviews In Biomedical Engineering 6: 21-23. 